\title{
COMPONENT GROUPS OF PURELY TORIC QUOTIENTS
}

\author{
Brian Conrad and William A. Stein
}

\begin{abstract}
Suppose $\pi: J \rightarrow A$ is an optimal quotient of abelian varieties over a $p$-adic field, optimal in the sense that $\operatorname{ker}(\pi)$ is connected. Assume that $J$ is equipped with a symmetric principal polarization $\theta$ (e.g., any Jacobian of a curve has such a polarization), that $J$ has semistable reduction, and that $A$ has purely toric reduction. In this paper, we express the group of connected components of the Néron model of $A$ in terms of the monodromy pairing on the character group of the torus associated to $J$. We apply our results in the case when $A$ is an optimal quotient of the modular Jacobian $J_{0}(N)$. For each prime $p$ that exactly divides $N$, we obtain an algorithm to compute the order of the component group of $A$ at $p$.
\end{abstract}

\section{Introduction}

Let $A$ be an abelian variety over the rational numbers $\mathbf{Q}$. Birch and Swinnerton-Dyer found a conjectural formula for the order of the ShafarevichTate group of $A$. The Tamagawa numbers $c_{p}$ of $A$ are among the quantities that appear in this formula. We now recall the definition of the Tamagawa numbers of an abelian variety (the definition of Néron model and component groups is given in Section 2).

Definition 1.1 (Tamagawa number). Let $p$ be a prime, let $\mathcal{A}$ be the Néron model of $A$ over the $p$-adic integers $\mathbf{Z}_{p}$, and let $\Phi_{A, p}$ be the component group of $\mathcal{A}$ at $p$. Then the Tamagawa number $c_{p}$ of $A$ at $p$ is the order of the subgroup $\Phi_{A, p}\left(\mathbf{F}_{p}\right)$ of $\mathbf{F}_{p}$-rational points in $\Phi_{A, p}\left(\overline{\mathbf{F}}_{p}\right)$.

Remark 1.2. The Tamagawa number is defined in a different way in some other papers, but the definitions are equivalent.

When $A$ has dimension one, $A$ is called an elliptic curve, and $A$ can be defined by a Weierstrass equation $y^{2}=x^{3}+a x+b$. Using that elliptic curves (and their related integral models) can be described by simple equations, Tate found an efficient algorithm to compute all of the Tamagawa numbers of $A$ (see [18]). In the case when $A$ is the Jacobian of a genus 2 curve, [7] discusses a method for computing the Tamagawa numbers of $A$. In this paper, we consider the situation in which $A$ has purely toric reduction at $p$, with no constraint on the dimension of $A$. For such $A$ we give an explicit description of the order of the group of connected components of the closed fiber of the Néron model

Received April 20, 2001.

Both authors were partially supported by the NSF and the Clay Mathematics Institute. 
of $A$. In the case when $A=A_{f}$ is a quotient of $J_{0}(N)$ attached to a newform $f \in S_{2}\left(\Gamma_{0}(N)\right)$ and $p \| N$, our method is completely explicit, and yields an algorithm to compute the Tamagawa number $c_{p}$ of $A$ (up to a bounded power of 2 ).

This paper is structured as follows. In Sections 2-6 we state and prove an explicit formula involving component groups of fairly general abelian varieties. Then in Section 7 we turn to quotients of modular Jacobians $J_{0}(N)$. We give some tables and discuss the arithmetic of quotients of $J_{0}(N)$ when $N$ is prime. In Section 8 we prove a couple of facts about toric reduction that are used in the proof of Theorem 6.1.

\section{The Main Results}

In this section, we summarize the main contributions of this paper. First we recall the precise definition of the component group of an abelian variety, then we state our main theorem.

Let $R$ be a discrete valuation ring with field of fractions $K$ and maximal ideal $\mathfrak{m}$, and let $k=R / \mathfrak{m}$ be the residue class field. Let $A$ be an abelian variety over $K$.

Definition 2.1 (Néron model). A Néron model of $A$ is a smooth commutative group scheme $\mathcal{A}$ over $R$ such that $A$ is its generic fiber and $\mathcal{A}$ satisfies the Néron mapping property: the restriction map

$$
\operatorname{Hom}_{R}(S, \mathcal{A}) \longrightarrow \operatorname{Hom}_{K}\left(S_{K}, A\right)
$$

is bijective for all smooth schemes $S$ over $R$.

The Néron mapping property implies that $\mathcal{A}$ is unique up to a unique isomorphism, so we will refer without hesitation to "the" Néron model of $A$. Néron models are separated and of finite type as opposed to just locally of finite type, even though their universal property is on the category of arbitrary smooth $R$-schemes. For more about Néron models see [2].

The closed fiber $\mathcal{A}_{k}$ of $\mathcal{A}$ is a group scheme over $k$, which need not be connected. Denote by $\mathcal{A}_{k}^{0}$ the connected component of $\mathcal{A}_{k}$ that contains the identity. We have an exact sequence

$$
0 \longrightarrow \mathcal{A}_{k}^{0} \longrightarrow \mathcal{A}_{k} \longrightarrow \Phi_{A} \longrightarrow 0
$$

where $\Phi_{A}$ is a finite étale group scheme over $k$. Equivalently, $\Phi_{A}$ is a commutative finite group equipped with a continuous action of $\operatorname{Gal}(\bar{k} / k)$.

Definition 2.2 (Component group). The component group of an abelian variety $A$ over $K$ is the group scheme $\Phi_{A}=\mathcal{A}_{k} / \mathcal{A}_{k}^{0}$. 
2.1. Statement of the Theorem. We now state our main result, supressing some of the definitions of the terms used until later (see Section 6 below for a more complete statement and the proof). Let $K$ be as above, and suppose $\pi: J \rightarrow A$ is an optimal quotient. Assume that $J$ is equipped with a symmetric principal polarization $\lambda$, in the sense of Definition 5.1. For example, the $\theta$ polarization of the Jacobian of a curve is a symmetric principal polarization. Also assume that $J$ has semistable reduction, and that $A$ has purely toric reduction.

We express the component group of $A$ in terms of the monodromy pairing associated to $J$. Let $m_{A}=\sqrt{\operatorname{deg}\left(\theta_{A}\right)}$, where $\theta_{A}: A^{\vee} \rightarrow A$ is induced by the principal polarization $\lambda$. Let $X_{J}$ be the character group of the toric part of the closed fiber of the Néron model of $J$. Let $\mathcal{L}$ be the saturation of the image of $X_{A}$ in $X_{J}$. The monodromy pairing induces a map $\alpha: X_{J} \rightarrow \operatorname{Hom}(\mathcal{L}, \mathbf{Z})$. Let $\Phi_{X}$ be the cokernel of $\alpha$ and $m_{X}=\left[\alpha\left(X_{J}\right): \alpha(\mathcal{L})\right]$ be the order of the finite group $\alpha\left(X_{J}\right) / \alpha(\mathcal{L})$. The main result of this paper is that

$$
\frac{\# \Phi_{A}}{m_{A}}=\frac{\# \Phi_{X}}{m_{X}},
$$

and this is recorded as Theorem 6.1 below.

Using the snake lemma, one sees that $\Phi_{X}$ is isomorphic to the image of the natural map $\Phi_{J} \rightarrow \Phi_{A}$, and the above formula implies that the cokernel of the map $\Phi_{J} \rightarrow \Phi_{A}$ has order $m_{A} / m_{X}$. A non-obvious consequence of this is that $m_{X} \mid m_{A}$.

In the context of modular forms, if the optimal quotient $J \rightarrow A$ arises from a newform on $\Gamma_{0}(N)$, then the quantities $m_{A}, m_{X}$ and $\Phi_{X}$ can be explicitly computed, hence we can compute $\# \Phi_{A}$. Note that the authors have not computed the structure of $\Phi_{A}$ as a group.

\section{Optimal Quotients}

Let $K$ be as in Section 2, let $J$ be an abelian variety equipped with a symmetric principal polarization $\theta_{J}$ (see Definition 5.1). For example, $J$ could be the Jacobian of a curve equipped with the canonical principal polarization arising from the $\theta$-divisor.

Definition 3.1 (Optimal quotient). An optimal quotient of $J$ is an abelian variety $A$ and a smooth surjective morphism $\pi: J \rightarrow A$ whose kernel is connected (i.e., an abelian variety).

Remark 3.2. Any connected scheme of finite type over a field is geometrically connected if it contains a rational point (e.g., if it is a group scheme). See [8, $\left.\mathrm{IV}_{2}, \S 4.5 .13\right]$.

Let $\pi: J \rightarrow A$ be an optimal quotient. Denote by $J^{\vee}$ and $A^{\vee}$ the abelian varieties dual to $J$ and $A$, respectively. Upon composing the dual of $\pi$ with $\theta_{J}^{\vee}=\theta_{J}$, we obtain a map

$$
A^{\vee} \stackrel{\pi^{\vee}}{\longrightarrow} J^{\vee} \stackrel{\theta_{J}}{\longrightarrow} J
$$


Proposition 3.3. The map $\theta_{J} \circ \pi^{\vee}: A^{\vee} \rightarrow J$ is a closed immersion.

Proof. Since $\theta_{J}$ is an isomorphism, we want to prove that $\pi^{\vee}$ is a closed immersion. It is a general fact that duals to surjections of abelian varieties with abelian variety kernel are closed immersions, but for lack of an adequate reference we recall the proof. Since a monomorphism between smooth finite type group schemes over a field is necessarily a closed immersion, it suffices to show that the commutative proper group scheme $\operatorname{ker}\left(\pi^{\vee}\right)$ vanishes. Since a non-zero commutative proper group scheme $G$ over a field $F$ necessarily has a non-zero finite subgroup scheme $G[n]$ for some $n$ (since either $\left(G_{/ F}\right)_{\text {red }}^{0}$ is an abelian variety or else $G$ is finite and non-zero), it suffices to show that $\operatorname{ker}\left(\pi^{\vee}\right)[n]$ vanishes for all positive integers $n$. In other words, it suffices to show that the induced map $A^{\vee}[n] \rightarrow J^{\vee}[n]$ is a closed immersion for all $n$.

Since Cartier duality interchanges faithfully flat maps and closed immersions, and the scheme-theoretic Weil pairing identifies the Cartier dual of the map induced by $\pi^{\vee}$ on $n$-torsion with $\pi: J[n] \rightarrow A[n]$, we just have to show that these latter maps are faithfully flat for all integers $n$. Using the short exact sequence

$$
0 \rightarrow \operatorname{ker}(\pi) \rightarrow J \rightarrow A \rightarrow 0
$$

in the abelian category of fppf abelian sheaves over $\operatorname{Spec}(K)$, the snake lemma gives an exact sequence

$$
0 \rightarrow \operatorname{ker}(\pi)[n] \rightarrow J[n] \rightarrow A[n] \rightarrow 0
$$

because $n: \operatorname{ker}(\pi) \rightarrow \operatorname{ker}(\pi)$ is a faithfully flat map (hence fppf surjective), as $\operatorname{ker}(\pi)$ is an abelian variety. This gives an isomorphism of group schemes

$$
J[n] / \operatorname{ker}(\pi)[n] \simeq A[n]
$$

compatible with the maps from $J[n]$, whence $J[n] \rightarrow A[n]$ is faithfully flat.

Henceforth we will abuse notation and denote the injection $A^{\vee} \rightarrow J$ by $\pi^{\vee}$. We define $\theta_{A}$ to be the composite $\pi \circ \pi^{\vee}$, so the kernel of $\theta_{A}$ equals the schemetheoretic intersection of $A^{\vee}$ and $B=\operatorname{ker}(\pi)$, as depicted in the following diagram:

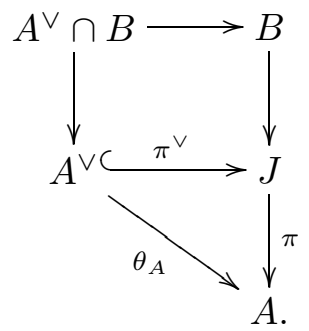

Since $\theta_{A}$ is a polarization (due to how its definition uses the polarization $\theta_{J}$ ) the degree of $\theta_{A}$ is a perfect square (see $[16, \S 16$, p. 150]).

Definition 3.4 (Degree). Define the degree of $A$ as a quotient of $J$ to be the integer

$$
m_{A}=\sqrt{\# \operatorname{ker}\left(\theta_{A}\right)} \text {. }
$$




\section{The Closed Fiber of the Néron Model}

In this section we recall some terminology associated with closed fibers of Néron models. Let $K, R$, and $k$ be as in Section 2 , and let $\Phi_{A}=\mathcal{A}_{k} / \mathcal{A}_{k}^{0}$ be the group scheme of connected components of the closed fiber $\mathcal{A}_{k}$. By Chevalley's structure theorem (see [3], or [4] for a modern account), if $K$ is a perfect extension field of $k$ (e.g., $K=\bar{k}$ ) then there is a unique short exact sequence

$$
0 \rightarrow \mathcal{C} \rightarrow \mathcal{A}_{K}^{0} \rightarrow \mathcal{B} \rightarrow 0
$$

with $\mathcal{C}$ a smooth affine algebraic $K$-group and $\mathcal{B}$ an abelian variety. Moreover, there is a unique exact sequence

$$
0 \rightarrow \mathcal{T} \rightarrow \mathcal{C} \rightarrow \mathcal{U} \rightarrow 0
$$

with $\mathcal{T}$ a torus and $\mathcal{U}$ unipotent.

Using the rigidity of tori, one can show that $\mathcal{T}$ is induced by a unique torus in $\mathcal{A}_{k}^{0}$. In particular, the condition that $\mathcal{B}=\mathcal{U}=0$ is equivalent to the condition that $\mathcal{A}_{k}^{0}$ be a torus, and the condition that $\mathcal{U}=0$ is equivalent to the condition that $\mathcal{A}_{k}^{0}$ be the extension of an abelian variety by a torus (i.e., be a semi-abelian variety). These conditions can be checked on a geometric closed fiber.

Definition 4.1. The abelian variety $A$ is said to have purely toric reduction if $\mathcal{A}_{k}^{0}$ is torus, and to have semistable reduction if $\mathcal{A}_{k}^{0}$ is a semi-abelian variety (i.e., $\mathcal{A}_{k}^{0}$ has vanishing unipotent part).

\subsection{The Monodromy Pairing on the Character Group.}

Definition 4.2 (Character group of torus). The character group

$$
X_{A}=\operatorname{Hom}_{\bar{k}}\left(\mathcal{T}_{\bar{k}}, \mathbf{G}_{m \bar{k}}\right)
$$

is a free abelian group contravariantly associated to $A$.

As discussed in [9], if $A$ is semistable there is a monodromy pairing $X_{A} \times$ $X_{A} \vee \mathbf{Z}$ and an exact sequence

$$
0 \rightarrow X_{A \vee} \rightarrow \operatorname{Hom}\left(X_{A}, \mathbf{Z}\right) \rightarrow \Phi_{A} \rightarrow 0 .
$$

Also, the canonical isomorphism $\left(A^{\vee}\right)^{\vee} \cong A$ induces an isomorphism

$$
X_{A \vee} \times X_{\left(A^{\vee}\right) \vee} \cong X_{A} \times X_{A^{\vee}},
$$

which identifies the monodromy pairing associated to $A^{\vee}$ with that associated to $A$.

Example 4.3 (Tate curve). Suppose $E=\mathbf{G}_{m} / q^{\mathbf{Z}}$ is a Tate curve over $\mathbf{Q}_{p}^{\mathrm{ur}}$. The monodromy pairing on $X_{E}=q^{\mathbf{Z}}$ is

$$
\langle q, q\rangle=\operatorname{ord}_{p}(q)=-\operatorname{ord}_{p}(j) .
$$

Thus $\Phi_{E}$ is cyclic of order $-\operatorname{ord}_{p}(j)$. 
Suppose $J$ is an abelian variety equipped with a symmetric principal polarization. Since $J$ is self dual via the given symmetric principal polarization, we can view the monodromy pairing on $J$ as a pairing $X_{J} \times X_{J} \rightarrow \mathbf{Z}$. Because the principal polarization on $J$ is symmetric the resulting pairing $X_{J} \times X_{J} \rightarrow \mathbf{Z}$ is symmetric, so there is no ambiguity about left versus right definitions of $X_{J} \rightarrow \operatorname{Hom}\left(X_{J}, \mathbf{Z}\right)$. The above exact sequence then becomes

$$
0 \rightarrow X_{J} \rightarrow \operatorname{Hom}\left(X_{J}, \mathbf{Z}\right) \rightarrow \Phi_{J} \rightarrow 0 \text {. }
$$

\section{The Degree of a Symmetric Isogeny}

We next relate the degree of the isogeny $A^{\vee} \rightarrow A$ defined at the end of Section 3 to the order of the cokernel of the induced map on the character groups of tori defined in Section 4.1. Let $K$ be as in Section 2, and let $A$ be an abelian variety over $K$.

Definition 5.1 (Symmetric isogeny). A symmetric isogeny $\varphi: A^{\vee} \rightarrow A$ is an isogeny such that the map

$$
\varphi^{\vee}: A^{\vee} \rightarrow\left(A^{\vee}\right)^{\vee}=A
$$

is equal to $\varphi$.

If $J$ and $A$ are as in Section 3 then the principal polarization $\theta_{J}$ of $J$ is symmetric, so the natural map $A^{\vee} \rightarrow A$ is a symmetric isogeny.

Lemma 5.2. Suppose that $A$ is a purely toric abelian variety over $K$ and that $\varphi: A^{\vee} \rightarrow A$ is a symmetric isogeny. Let $\varphi_{a}: X_{A} \rightarrow X_{A^{\vee}}$ denote the induced map on character groups. Then

$$
\operatorname{deg}(\varphi)=\# \operatorname{coker}\left(\varphi_{a}\right)^{2} .
$$

Proof. By Corollary 8.7 applied to our isogeny $\varphi$ (so what we are presently calling $A^{\vee}$ and $A$ are respectively called $A$ and $B$ in the discussion surrounding Theorem 8.6), we deduce that

$$
\operatorname{deg}(\varphi)=\# \operatorname{ker}(\varphi)=\# \operatorname{ker}\left(\varphi_{t}\right) \cdot \# \operatorname{ker}\left(\varphi_{t}^{\vee}\right)
$$

where $\varphi_{t}$ and $\varphi_{t}^{\vee}$ are the maps induced by $\varphi$ and $\varphi^{\vee}$ on closed fiber tori.

Since the character group $X_{A}$ is, by definition, $\operatorname{Hom}_{\bar{k}}\left(\mathcal{T}_{\bar{k}}, \mathbf{G}_{m \bar{k}}\right)$, where $\mathcal{T}$ is the toric part of the closed fiber of $A$, it follows that \# $\operatorname{ker}\left(\varphi_{t}\right)=\# \operatorname{coker}\left(\varphi_{a}\right)$. Since $\varphi=\varphi^{\vee}$, this proves the lemma.

\section{Statement and Proof of the Main Theorem}

Let $K$ be as in Section 2, and let $\pi: J \rightarrow A$ be an optimal quotient. Assume that $J$ is equipped with a symmetric principal polarization $\lambda$, that $J$ has semistable reduction, and that $A$ has purely toric reduction. Let $X_{A}, X_{A^{\vee}}$, and $X_{J}$ denote the character groups of the toric parts of the closed fibers of the abelian varieties $A, A^{\vee}$, and $J$, respectively. 
Let $\pi: J \rightarrow A$ be an optimal quotient, and let $\theta: A^{\vee} \rightarrow A$ denote the induced polarization. Let $\pi_{*}, \pi^{*}, \theta_{*}$, and $\theta^{*}$ be the maps induced on character groups by the various functorialities, as indicated in the following two key diagrams:
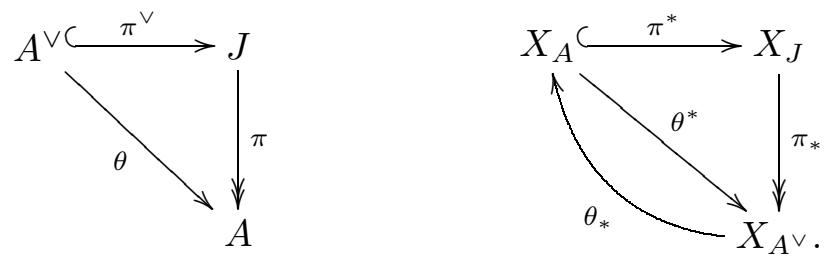

The surjectivity of $\pi_{*}$ is proved in Theorem 8.2. The injectivity of $\pi^{*}$ follows because

$$
\theta_{*} \pi_{*} \pi^{*}=\theta_{*} \theta^{*}=\operatorname{deg}(\theta) \neq 0,
$$

and multiplication by a nonzero integer on a free abelian group is injective.

Let $\mathcal{L}$ be the saturation of $\pi^{*} X_{A}$ in $X_{J}$; thus $\pi^{*} X_{A}$ is a finite-index subgroup of $\mathcal{L}$ and the quotient $X_{J} / \mathcal{L}$ is torsion free. Let

$$
\alpha: X_{J} \rightarrow \operatorname{Hom}\left(\pi^{*} X_{A}, \mathbf{Z}\right)
$$

be the map defined by the monodromy pairing restricted to $X_{J} \times \pi^{*} X_{A}$. For $L$ of finite index in $\mathcal{L}$, define the degree of $L$ to be

$$
m_{L}=\left[\alpha\left(X_{J}\right): \alpha(L)\right]
$$

and the component group of $L$ to be

$$
\Phi_{L}=\operatorname{coker}\left(X_{J} \rightarrow \operatorname{Hom}(L, \mathbf{Z})\right) .
$$

When $L=\mathcal{L}$ and $A$ is fixed, for simplicity we write $m_{X}=m_{\mathcal{L}}$ and $\Phi_{X}=\Phi_{\mathcal{L}}$.

Recall that $\Phi_{A}$ is the component group of $A$ and $m_{A}$ is the square root of the degree of the induced map $A^{\vee} \rightarrow A$.

Theorem 6.1. For any subgroup $L$ of finite index in $\mathcal{L}$, the following relation holds:

$$
\frac{\# \Phi_{A}}{m_{A}}=\frac{\# \Phi_{L}}{m_{L}} .
$$

6.1. Proof of the Main Theorem. The notation in this section is as in previous section.

Lemma 6.2. Let $\pi_{*}: X_{J} \rightarrow X_{A^{\vee}}$ and $\alpha: X_{J} \rightarrow \operatorname{Hom}\left(\pi^{*} X_{A}, \mathbf{Z}\right)$ be as in previous section. Then

$$
\operatorname{ker}\left(\pi_{*}\right)=\operatorname{ker}(\alpha) .
$$

Proof. Suppose $x \in \operatorname{ker}\left(\pi_{*}\right)$, and let $y=\pi^{*} z$ with $z \in X_{A}$. Then

$$
\langle x, y\rangle=\left\langle x, \pi^{*} z\right\rangle=\left\langle\pi_{*} x, z\right\rangle=0,
$$

so $x \in \operatorname{ker}(\alpha)$. Next let $x \in \operatorname{ker}(\alpha)$. Then for all $z \in X_{A}$,

$$
0=\left\langle x, \pi^{*} z\right\rangle=\left\langle\pi_{*} x, z\right\rangle,
$$


so $\pi_{*} x$ is in the kernel of the monodromy map

$$
X_{A^{\vee}} \rightarrow \operatorname{Hom}\left(X_{A}, \mathbf{Z}\right) .
$$

Since $X_{A} \vee$ and $\operatorname{Hom}\left(X_{A}, \mathbf{Z}\right)$ are free of the same finite rank and the cokernel is torsion, the monodromy map is injective. Thus $\pi_{*} x=0$ and $x \in \operatorname{ker}\left(\pi_{*}\right)$.

Let $\pi^{*}: X_{A} \rightarrow X_{J}$ be as in previous section.

Lemma 6.3. The monodromy-pairing map $X_{J} \rightarrow \operatorname{Hom}\left(X_{J}, \mathbf{Z}\right)$ composed with restriction $\operatorname{Hom}\left(X_{J}, \mathbf{Z}\right) \rightarrow \operatorname{Hom}\left(\pi^{*} X_{A}, \mathbf{Z}\right)$ gives rise to an exact sequence

$$
X_{J} \rightarrow \operatorname{Hom}\left(\pi^{*} X_{A}, \mathbf{Z}\right) \rightarrow \Phi_{A} \rightarrow 0 .
$$

Proof. Lemma 6.2 gives the following commutative diagram with exact rows

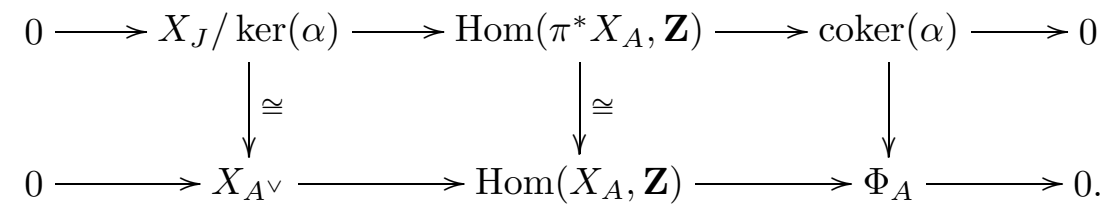

By Lemma 6.2, the first vertical map is an isomorphism. The second is an isomorphism because it is induced by the isomorphism $\pi^{*}: X_{A} \rightarrow \pi^{*} X_{A}$. It follows that $\operatorname{coker}(\alpha) \cong \Phi_{A}$, as claimed.

Recall that $\mathcal{L}$ denotes the saturation of $\pi^{*} X_{A}$ in $X_{J}$, and that $L \subset \mathcal{L}$ denotes a subgroup of finite index.

Lemma 6.4. The rational number $\frac{\# \Phi_{L}}{m_{L}}$ is independent of the choice of $L$.

Proof. Suppose $L^{\prime}$ is another finite index subgroup of $\mathcal{L}$, and let $n=\left[L: L^{\prime}\right]$. Here $n$ is a rational number, the lattice index of $L^{\prime}$ in $L$. Since $\alpha$ is injective when restricted to $\mathcal{L}$, it follows that

$$
m_{L^{\prime}}=\left[\alpha\left(X_{J}\right): \alpha\left(L^{\prime}\right)\right]=\left[\alpha\left(X_{J}\right): \alpha(L)\right] \cdot\left[\alpha(L): \alpha\left(L^{\prime}\right)\right]=m_{L} \cdot n .
$$

Similarly, $\# \Phi_{L^{\prime}}=\# \Phi_{L} \cdot n$.

$$
\begin{aligned}
& \text { Recall that } m_{A}=\sqrt{\operatorname{deg}(\theta)} \text { and } \\
& \qquad \Phi_{A} \cong \operatorname{coker}\left(X_{A} \vee \rightarrow \operatorname{Hom}\left(X_{A}, \mathbf{Z}\right)\right),
\end{aligned}
$$

where $m_{A}$ is the degree of $A$ and $\Phi_{A}$ is the component group of $A$.

Proof of Theorem 6.1. By Lemma 6.4 we may assume that $L=\pi^{*} X_{A}$. With this choice of $L$, Lemma 6.3 asserts that $\Phi_{L} \cong \Phi_{A}$. By Lemma 6.2, properties 
of the index, and Lemma 5.2 we have

$$
\begin{aligned}
m_{L} & =\left[\alpha\left(X_{J}\right): \alpha(L)\right] \\
& =\left[\pi_{*}\left(X_{J}\right): \pi_{*}(L)\right] \\
& =\left[X_{A}: \pi_{*}\left(\pi^{*} X_{A}\right)\right] \\
& =\left[X_{A}: \theta^{*} X_{A}\right] \\
& =\# \operatorname{coker}\left(\theta^{*}\right) \\
& =\sqrt{\operatorname{deg}(\theta)}=m_{A} .
\end{aligned}
$$

Recall that $\Phi_{\mathcal{L}}$ denotes the cokernel of the natural map $X_{J} \rightarrow \operatorname{Hom}(\mathcal{L}, \mathbf{Z})$ induced by composing the monodromy map $X_{J} \rightarrow \operatorname{Hom}\left(X_{J}, \mathbf{Z}\right)$ with the natural restriction map $\operatorname{Hom}\left(X_{J}, \mathbf{Z}\right) \rightarrow \operatorname{Hom}(\mathcal{L}, \mathbf{Z})$.

Proposition 6.5. The group $\Phi_{\mathcal{L}}$ is canonically isomorphic to the image of the map from $\Phi_{J}$ to $\Phi_{A}$ induced by $\pi: J \rightarrow A$. Thus

$$
\operatorname{image}\left(\Phi_{J} \rightarrow \Phi_{A}\right) \cong \Phi_{\mathcal{L}}
$$

Proof. Since $\pi^{*} X_{A} \subset \mathcal{L} \subset X_{J}$, an application of Lemma 6.3 gives the following commutative diagram with exact rows:

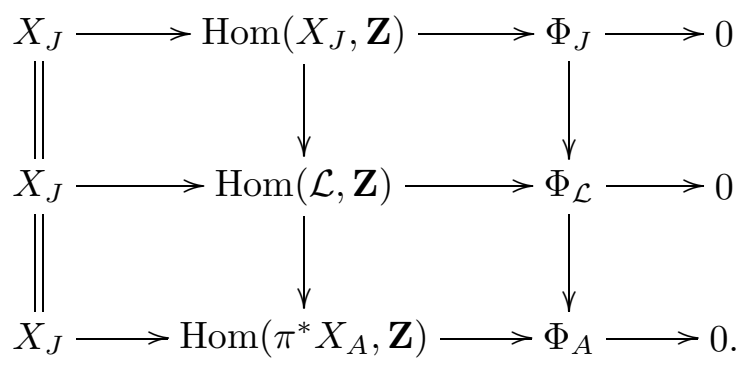

The map $\operatorname{Hom}(\mathcal{L}, \mathbf{Z}) \rightarrow \operatorname{Hom}\left(\pi^{*} X_{A}, \mathbf{Z}\right)$ is an isomorphism, so the map $\Phi_{\mathcal{L}} \rightarrow \Phi_{A}$ is injective. Thus

$$
\operatorname{image}\left(\Phi_{J} \rightarrow \Phi_{A}\right) \cong \operatorname{image}\left(\Phi_{J} \rightarrow \Phi_{\mathcal{L}}\right)
$$

The cokernel of $\operatorname{Hom}\left(X_{J}, \mathbf{Z}\right) \rightarrow \operatorname{Hom}(\mathcal{L}, \mathbf{Z})$ surjects onto the cokernel of $\Phi_{J} \rightarrow$ $\Phi_{\mathcal{L}}$. Using the exact sequence

$$
0 \rightarrow \mathcal{L} \rightarrow X_{J} \rightarrow X_{J} / \mathcal{L} \rightarrow 0
$$

we find that

$$
\operatorname{coker}\left(\operatorname{Hom}\left(X_{J}, \mathbf{Z}\right) \rightarrow \operatorname{Hom}(\mathcal{L}, \mathbf{Z})\right) \subset \operatorname{Ext}^{1}\left(X_{J} / \mathcal{L}, \mathbf{Z}\right) .
$$

Because $\mathcal{L}$ is saturated, the quotient $X_{J} / \mathcal{L}$ is torsion free, so the indicated Ext ${ }^{1}$ group vanishes. Thus the map $\Phi_{J} \rightarrow \Phi_{\mathcal{L}}$ is surjective, from which the proposition follows. 
Corollary 6.6. The cokernel of the map from $\Phi_{J}$ to $\Phi_{A}$ induced by $\pi: J \rightarrow A$ has order $m_{A} / m_{\mathcal{L}}$. Thus

$$
\# \operatorname{coker}\left(\Phi_{J} \rightarrow \Phi_{A}\right)=\frac{m_{A}}{m_{\mathcal{L}}}
$$

Proof. Combine Theorem 6.1 and Proposition 6.5.

\section{Optimal Quotients of $J_{0}(N)$}

In this section we specialize the general results of the rest of this paper to the concrete case in which $J=J_{0}(N)$ is the Jacobian of a modular curve, and $A=A_{f}$ is an optimal quotient of $J$ attached to a modular forms. The paper [12] contains more computations like these.

7.1. Modular Curves and Semistable Reduction. Let $X_{0}(N)$ be the modular curve associated to the subgroup $\Gamma_{0}(N)$ of $\mathrm{SL}_{2}(\mathbf{Z})$ that consists of those matrices which are upper triangular modulo $N$. The algebraic curve $X_{0}(N)_{\mathbf{C}}$ can be constructed as a Riemann surface as the quotient

$$
\Gamma_{0}(N) \backslash\left(\{z: z \in \mathbf{C}, \operatorname{Im}(z)>0\} \cup \mathbf{P}^{1}(\mathbf{Q})\right),
$$

and $X_{0}(N)$ has a canonical structure of algebraic curve over $\mathbf{Q}$.

It is well known that the $p$-new part of the Jacobian $J_{0}(N)$ of $X_{0}(N)$ has purely toric reduction at $p$ when $p \| N$. Let us briefly recall the reason, writing $N=M p$. Using the description of closed fibers of modular curves [10, Ch. 13] and Raynaud's result relating Néron models and Picard functors (as summarized in $\left[2\right.$, Ch. 9]), the standard finite flat degeneracy maps $X_{0}(M p) \rightarrow X_{0}(M)$ over $\mathbf{Z}_{(p)}$ induce a "pushfoward" map on Néron model connected components

$$
\operatorname{Pic}_{X_{0}(M p) / \mathbf{z}_{(p)}}^{0} \longrightarrow \operatorname{Pic}_{X_{0}(M) / \mathbf{z}_{(p)}}^{0} \times \operatorname{Pic}_{X_{0}(M) / \mathbf{z}_{(p)}}^{0}
$$

which on the closed fiber is the map induced by pullback to the two components $X_{0}(M) / \mathbf{F}_{p}$ in $X_{0}(M p) / \mathbf{F}_{p}$. The kernel of this latter map is a torus [2, Ex. 9.2.8], yet this kernel is visibly isogenous to the semistable mod $p$ fiber of the dual of $J_{0}(M p)^{\text {new }}$, whence the purely toric conclusion.

7.2. Newforms and Optimal Quotients. The Hecke algebra

$$
\mathbf{T}=\mathbf{Z}\left[\ldots T_{n} \ldots\right] \subset \operatorname{End}\left(J_{0}(N)\right)
$$

is a commutative ring of endomorphisms of $J_{0}(N)$ of $\mathbf{Z}$-rank equal to the dimension of $J_{0}(N)$. The character group $X_{J, p}$ of $J_{0}(N)$ at $p$ is equipped with a functorial action of $\mathbf{T}$. The Hecke algebra $\mathbf{T}$ also acts on the complex vector space $S=S_{2}\left(\Gamma_{0}(N), \mathbf{C}\right)$ of cusp forms.

Let $f$ be a newform, and associate to $f$ the ideal $I_{f}$ of the Hecke algebra $\mathbf{T}$ of elements which annihilate $f$. Then $\mathcal{O}_{f}=\mathbf{T} / I_{f}$ is an order in the ring of integers of the totally real number field $K_{f}$ obtained by adjoining the Fourier coefficients of $f$ to $\mathbf{Q}$. The quotient

$$
A_{f}=J_{0}(N) / I_{f} J_{0}(N)
$$


is an optimal quotient of $J_{0}(N)$ of dimension equal to $\left[K_{f}: \mathbf{Q}\right]$. As discussed in the previous section, $A_{f}$ is purely toric at $p$.

7.3. Tamagawa Numbers. Let Frob ${ }_{p} X_{J} \rightarrow X_{J}$ denote the map induced by the Frobenius automorphism. We have $\operatorname{Frob}_{p}=-W_{p}$, where $W_{p}$ is the map induced by the Atkin-Lehner involution on $J_{0}(p)$. Let $f$ be a newform, $A=A_{f}$ the corresponding optimal quotient, and $w_{p}$ the sign of the eigenvalue of $W_{p}$ on $f$.

\section{Proposition 7.1.}

$$
\Phi_{A}\left(\mathbf{F}_{p}\right)= \begin{cases}\Phi_{A}\left(\overline{\mathbf{F}}_{p}\right) & \text { if } w_{p}=-1 \\ \Phi_{A}\left(\overline{\mathbf{F}}_{p}\right)[2] & \text { if } w_{p}=1\end{cases}
$$

Proof. If $w_{p}=-1$, then $\operatorname{Frob}_{p}=1$ and the $\operatorname{Gal}\left(\overline{\mathbf{F}}_{p} / \mathbf{F}_{p}\right)$-action of $\Phi_{A}\left(\overline{\mathbf{F}}_{p}\right)$ is trivial. In this case $\Phi\left(\mathbf{F}_{p}\right)=\Phi\left(\overline{\mathbf{F}}_{p}\right)$. Next suppose $w_{p}=1$. Recall that we have an exact sequence

$$
0 \rightarrow X_{A^{\vee}} \rightarrow \operatorname{Hom}\left(X_{A}, \mathbf{Z}\right) \rightarrow \Phi_{A} \rightarrow 0 .
$$

Since $W_{p}$ acts as +1 on $f$, it also acts as +1 on each of the modules $A, X_{A}$, $\operatorname{Hom}\left(X_{A}, \mathbf{Z}\right)$, and $\Phi_{A}$. Thus $\operatorname{Frob}_{p}=-W_{p}$ acts as -1 on $\Phi_{A}$. Since the subgroup of 2-torsion elements of a finite abelian group equals the subgroup of elements fixed under -1 , it follows that $\Phi_{A}\left(\mathbf{F}_{p}\right)=\Phi_{A}\left(\overline{\mathbf{F}}_{p}\right)[2]$.

Warning. When extending this result to the whole of $J_{0}(N)$, be careful. The action of $\mathrm{Frob}_{p}=T_{p}$ need not be by \pm 1 , even though it must be by an involution of order 2. For example, the component group of $J_{0}(65)$ at 5 is cyclic of order 42. The action of Frob $b_{5}$ is by multiplication by -13 . Note that $(-13)^{2}=1$ $(\bmod 42)$. The fixed points of multiplication by -13 is the order 14 subgroup of $\mathbf{Z} / 42 \mathbf{Z}$.

7.4. Computing Component Groups. Using modular symbols, we can enumerate the optimal quotients $A_{f}$ of $J_{0}(N)$ (see, e.g., [1]) and compute the degree $m_{A}$ (see $[12, \S 3.1]$ ). Suppose $p$ is a prime that exactly divides $N$. As explained in [12], the method of graphs (see [14]) or the ideal theory of quaternion algebras (see [11]) can be used to compute $X=X_{J_{0}(N), p}$ with its $\mathbf{T}$-action and the monodromy pairing. We can then compute the following three modules:

1. the saturated submodule $\mathcal{L}=\bigcap_{t \in I_{f}} \operatorname{ker}(t)$ of $X$,

2 . the character group degree $m_{X}=m_{\mathcal{L}}$, and

3. $\Phi_{X}=\Phi_{\mathcal{L}}$.

By Theorem 6.1 we obtain

$$
\# \Phi_{A, p}=\# \Phi_{X} \cdot \frac{m_{A}}{m_{X}} .
$$


7.5. The Eisenstein Nature of Component Groups. The theorem below, which generalizes some of the results of [13] and [15], was conjectured by the second author after computing many component groups of quotients of $J_{0}(p)$ using the results of this paper. M. Emerton read an early version of this paper and subsequently announced a proof of the theorem below (see [6]).

Theorem 7.2 (Emerton). Let $p$ be a prime and let $f_{1}, \ldots, f_{n}$ be a set of representatives for the Galois-conjugacy classes of newforms in $S_{2}\left(\Gamma_{0}(p)\right)$. Let $A_{1}, \ldots, A_{n}$ be the optimal quotients associated to $f_{1}, \ldots, f_{n}$, respectively. Then for each $i, i=1, \ldots, n$, we have

$$
\# A_{i}(\mathbf{Q})_{\text {tor }}=\# \Phi_{A_{i}}\left(\overline{\mathbf{F}}_{p}\right)=\# \Phi_{A_{i}}\left(\mathbf{F}_{p}\right) \text {. }
$$

Furthermore,

$$
\# \Phi_{J_{0}(p)}\left(\overline{\mathbf{F}}_{p}\right)=\prod_{i=1}^{n} \# \Phi_{A_{i}}\left(\overline{\mathbf{F}}_{p}\right) .
$$

Before Emerton proved the above assertion, the second author verified it using the algorithm of this paper for all $p \leq 757$, and, up to a power of 2 , for all $p<2000$.

Remark 7.3. It is tempting to guess that, e.g., the natural map

$$
\Phi_{J_{0}(113)}\left(\overline{\mathbf{F}}_{113}\right) \rightarrow \prod_{i=1}^{4} \Phi_{A_{i}}\left(\overline{\mathbf{F}}_{113}\right)
$$

is an isomorphism, but this is incorrect. Two of the $\Phi_{A_{i}}\left(\overline{\mathbf{F}}_{113}\right)$ have order 2 , so the product $\prod_{i=1}^{4} \Phi_{A_{i}}\left(\overline{\mathbf{F}}_{113}\right)$ is not a cyclic group. However, Mazur proved that the groups $\Phi_{J_{0}(p)}\left(\overline{\mathbf{F}}_{p}\right)$ are cyclic for all primes $p$.

7.6. Examples. In this section we give some examples of the numbers involved in computing component groups of quotients of $J_{0}(N)$. For more examples, see [12]. We use the notation for abelian varieties that is described in [1]. For example, 65A is the "first" abelian variety quotient of $J_{0}(65)$ attached to a newform.

7.6.1. Quotients of $J_{0}(N)$. Table 1 contains many of the quantities involved in the computation of component groups for each of the newform optimal quotients for $N \in\{65,66,68,69\}$.

7.6.2. Quotients of $J_{0}(p)^{-}$. We computed the quantities $m_{A}, m_{X}$, and $\Phi_{X}$ for each abelian variety $A_{f}$ associated to a newform of prime level $p$ with $p \leq$ 631. Table 2 lists those $A_{f}$ for which $w_{p}=-1$, along with the order of the corresponding component group. The first column, which is labeled " $A$ " contains a description of $A_{f}$, the second column, labeled " $d$ ", contains the dimension of $A_{f}$, and the third column, labeled " $\# \Phi_{A}$ ", contains the order $\# \Phi_{A_{f}, p}\left(\overline{\mathbf{F}}_{p}\right)$ of the component group. 
TABLE 1. Component groups of quotients of $J_{0}(N)$

$\begin{array}{lccccccc}A & \operatorname{dim} & p & w_{p} & \# \Phi_{X} & m_{X} & m_{A} & \# \Phi_{A} \\ \mathbf{6 5 A} & 1 & 5 & + & 1 & 2 & 2 & 1 \\ & & 13 & + & 1 & 2 & & 1 \\ \mathbf{6 5 B} & 2 & 5 & + & 3 & 2^{2} & 2^{2} & 3 \\ & & 13 & - & 3 & 2^{2} & & 3 \\ \mathbf{6 5 C} & 2 & 5 & - & 7 & 2^{2} & 2^{2} & 7 \\ & & 13 & + & 1 & 2^{2} & & 1 \\ \mathbf{6 6 A} & 1 & 2 & + & 1 & 2 & 2^{2} & 2 \\ & & 3 & - & 3 & 2^{2} & & 3 \\ & & 11 & + & 1 & 2^{2} & & 1 \\ \mathbf{6 6 B} & 1 & 2 & - & 2 & 2 & 2^{2} & 2^{2} \\ & & 3 & + & 1 & 2^{2} & & 1 \\ & & 11 & + & 1 & 2^{2} & & 1 \\ \mathbf{6 6 C} & 1 & 2 & - & 1 & 2 & 2^{2} \cdot 5 & 2 \cdot 5 \\ & & 3 & - & 1 & 2^{2} & & 5 \\ & & 11 & - & 1 & 2^{2} \cdot 5 & & 1 \\ \mathbf{6 8 A} & 2 & 17 & + & 2 & 2 \cdot 3 & 2 \cdot 3 & 2 \\ & & & & & & & 2 \\ \mathbf{6 9 A} & 1 & 3 & - & 2 & 2 & 2 & 2 \\ & & 23 & + & 1 & 2 & & 1 \\ \mathbf{6 9 B} & 2 & 3 & + & 2 & 2 & 2 \cdot 11 & 2 \cdot 11 \\ & & 23 & - & 2 & 2 \cdot 11 & & 2\end{array}$

Remark 7.4. Theorem 7.2 together with [13, Prop. II.17.10] imply that the component groups of the $A_{f}$ for which $w_{p}=+1$ are trivial, so we do not list them. An optimal quotient $A_{f}$ of $J_{0}(p)$ with nonzero component group has nonzero rational torsion (by Theorem 7.2), so it factors through the Eisenstein quotient of $J_{0}(p)$. Also $w_{p}$ acts as -1 on the Eisenstein quotient of $J_{0}(p)$, which is [13, Prop. II.17.10], and which is a deep result because of subtleties at the prime 2 (see the discussion in [13, III.1]). 
TABle 2. Component groups of quotients of $J_{0}(p)^{-}$

\begin{tabular}{|c|c|c|c|c|c|c|c|c|c|c|c|}
\hline$A$ & $d$ & $\# \Phi_{A}$ & $A$ & $d$ & $\# \Phi_{A}$ & $A$ & $d$ & $\# \Phi_{A}$ & $A$ & $d$ & $\# \Phi_{A}$ \\
\hline $11 \mathrm{~A}$ & 1 & 5 & 157B & 7 & 13 & 313A & 2 & 1 & 487B & 2 & 3 \\
\hline $17 \mathrm{~A}$ & 1 & $2^{2}$ & $163 \mathrm{C}$ & 7 & $3^{3}$ & $313 \mathrm{C}$ & 12 & $2 \cdot 13$ & $487 \mathrm{C}$ & 3 & 1 \\
\hline 19A & 1 & 3 & 167B & 12 & 83 & 317B & 15 & 79 & $487 \mathrm{D}$ & 16 & $3^{3}$ \\
\hline $23 A$ & 2 & 11 & 173B & 10 & 43 & 331D & 16 & $5 \cdot 11$ & $491 \mathrm{C}$ & 29 & $5 \cdot 7^{2}$ \\
\hline 29A & 2 & 7 & 179A & 1 & 1 & 337B & 15 & $2^{2} \cdot 7$ & $499 \mathrm{C}$ & 23 & 83 \\
\hline $31 \mathrm{~A}$ & 2 & 5 & $179 \mathrm{C}$ & 11 & 89 & 347D & 19 & 173 & 503B & 1 & 1 \\
\hline 37B & 1 & 3 & 181B & 9 & $3 \cdot 5$ & 349B & 17 & 29 & $503 \mathrm{C}$ & 1 & 1 \\
\hline $41 \mathrm{~A}$ & 3 & $2 \cdot 5$ & 191B & 14 & $5 \cdot 19$ & 353A & 1 & 2 & 503D & 3 & 1 \\
\hline $43 B$ & 2 & 7 & $193 C$ & 8 & $2^{4}$ & 353B & 3 & 2 & $503 F$ & 26 & 251 \\
\hline $47 \mathrm{~A}$ & 4 & 23 & $197 \mathrm{C}$ & 10 & $7^{2}$ & 353D & 14 & $2 \cdot 11$ & $509 B$ & 28 & 127 \\
\hline 53B & 3 & 13 & 199A & 2 & 1 & 359D & 24 & 179 & 521B & 29 & $2 \cdot 5 \cdot 13$ \\
\hline $59 A$ & 5 & 29 & $199 \mathrm{C}$ & 10 & $3 \cdot 11$ & 367B & 19 & 61 & $523 \mathrm{C}$ & 26 & $3 \cdot 29$ \\
\hline $61 B$ & 3 & 5 & 211A & 2 & 5 & $373 \mathrm{C}$ & 17 & 31 & 541B & 24 & $3^{2} \cdot 5$ \\
\hline $67 \mathrm{~A}$ & 1 & 1 & 211D & 9 & 7 & 379B & 18 & $3^{2} \cdot 7$ & $547 \mathrm{C}$ & 25 & $7 \cdot 13$ \\
\hline $67 \mathrm{C}$ & 2 & 11 & $223 \mathrm{C}$ & 12 & 37 & $383 \mathrm{C}$ & 24 & 191 & $557 \mathrm{~B}$ & 1 & 1 \\
\hline $71 \mathrm{~A}$ & 3 & 5 & $227 B$ & 2 & 1 & 389A & 1 & 1 & $557 \mathrm{D}$ & 26 & 139 \\
\hline $71 B$ & 3 & 7 & $227 \mathrm{C}$ & 2 & 1 & 389E & 20 & 97 & $563 \mathrm{~A}$ & 1 & 1 \\
\hline $73 \mathrm{~A}$ & 1 & 2 & 227 E & 10 & 113 & 397B & 2 & 1 & $563 \mathrm{E}$ & 31 & 281 \\
\hline $73 \mathrm{C}$ & 2 & 3 & $229 \mathrm{C}$ & 11 & 19 & $397 \mathrm{C}$ & 5 & 11 & 569B & 31 & $2 \cdot 71$ \\
\hline 79B & 5 & 13 & 233A & 1 & 2 & 397D & 10 & 3 & 571A & 1 & 1 \\
\hline $83 B$ & 6 & 41 & $233 \mathrm{C}$ & 11 & 29 & 401B & 21 & $2^{2} \cdot 5^{2}$ & 571B & 1 & 1 \\
\hline 89B & 1 & 2 & 239B & 17 & $7 \cdot 17$ & 409B & 20 & $2 \cdot 17$ & $571 C$ & 2 & 1 \\
\hline $89 \mathrm{C}$ & 5 & 11 & 241B & 12 & $2^{2} \cdot 5$ & 419B & 26 & $11 \cdot 19$ & $571 D$ & 2 & 1 \\
\hline $97 \mathrm{~B}$ & 4 & $2^{3}$ & 251B & 17 & $5^{3}$ & 421B & 19 & $5 \cdot 7$ & $571 \mathrm{~F}$ & 4 & 1 \\
\hline 101B & 7 & $5^{2}$ & 257B & 14 & $2^{6}$ & 431B & 1 & 1 & $571 I$ & 18 & $5 \cdot 19$ \\
\hline 103B & 6 & 17 & 263B & 17 & 131 & 431D & 3 & 1 & $577 \mathrm{~A}$ & 2 & 3 \\
\hline 107B & 7 & 53 & $269 C$ & 16 & 67 & $431 \mathrm{~F}$ & 24 & $5 \cdot 43$ & 577B & 2 & 1 \\
\hline 109A & 1 & 1 & 271B & 16 & $3^{2} \cdot 5$ & 433A & 1 & 1 & $577 \mathrm{C}$ & 3 & 1 \\
\hline 109C & 4 & $3^{2}$ & 277B & 3 & 1 & 433B & 3 & 1 & $577 \mathrm{D}$ & 18 & $2^{4}$ \\
\hline 113A & 1 & 2 & 277D & 9 & 23 & 433D & 16 & $2^{2} \cdot 3^{2}$ & $587 \mathrm{C}$ & 31 & 293 \\
\hline 113B & 2 & 2 & 281B & 16 & $2 \cdot 5 \cdot 7$ & $439 \mathrm{C}$ & 25 & 73 & 593B & 1 & 2 \\
\hline 113D & 3 & 7 & 283B & 14 & 47 & $443 C$ & 1 & 1 & $593 \mathrm{C}$ & 2 & 1 \\
\hline 127B & 7 & $3 \cdot 7$ & 293B & 16 & 73 & $443 \mathrm{E}$ & 22 & $13 \cdot 17$ & 593E & 27 & $2 \cdot 37$ \\
\hline 131B & 10 & $5 \cdot 13$ & $307 \mathrm{~A}$ & 1 & 1 & 449B & 23 & $2^{4} \cdot 7$ & $599 \mathrm{C}$ & 37 & $13 \cdot 23$ \\
\hline 137B & 7 & $2 \cdot 17$ & 307B & 1 & 1 & $457 \mathrm{C}$ & 20 & $2 \cdot 19$ & $601 \mathrm{~B}$ & 29 & $2 \cdot 5^{2}$ \\
\hline 139A & 1 & 1 & $307 \mathrm{C}$ & 1 & 1 & 461D & 26 & $5 \cdot 23$ & $607 \mathrm{D}$ & 31 & 101 \\
\hline $139 \mathrm{C}$ & 7 & 23 & 307D & 1 & 1 & 463B & 22 & $7 \cdot 11$ & $613 \mathrm{C}$ & 27 & $3 \cdot 17$ \\
\hline 149B & 9 & 37 & $307 E$ & 2 & 3 & $467 \mathrm{C}$ & 26 & 233 & $617 \mathrm{~B}$ & 28 & $2 \cdot 7 \cdot 11$ \\
\hline 151B & 3 & 1 & $307 F$ & 9 & 17 & 479B & 32 & 239 & 619B & 30 & 103 \\
\hline $151 \mathrm{C}$ & 6 & $5^{2}$ & 311B & 22 & $5 \cdot 31$ & $487 \mathrm{~A}$ & 2 & 1 & 631B & 32 & $3 \cdot 5 \cdot 7$ \\
\hline
\end{tabular}

\section{Appendix: Some Facts Concerning Toric Reduction}

Let $R$ be a discrete valuation ring with fraction field $K$ and residue field $k$. For any abelian variety $A$ over $K$, with Néron model $\mathcal{A}$ over $R$, we denote by 
$X_{A}$ the character group of the toric part of $\mathcal{A}_{k}^{0}$ (the connected component of the closed fiber of $\mathcal{A}$ ). All group schemes below are understood to be commutative.

Our aim in this appendix is to prove a couple of facts (Theorem 8.2 and Theorem 8.6) which are no doubt well-known to experts but for which published proofs do not appear to be readily available. We begin with a simple and basic lemma.

Lemma 8.1. Let $f: G \rightarrow G^{\prime}$ be a map between multiplicative (resp. étale) finite flat group schemes over $R$. The map $f$ is a closed immersion (resp. faithfully flat) if and only if the generic fiber map $f_{K}$ is a closed immersion (resp. faithfully flat).

Proof. Cartier duality interchanges étaleness and multiplicativeness, as well as closed immersions and faithfully flat maps (as the latter two properties may be checked on the closed fiber, for which one is reduced to the standard case of finite commutative group schemes over a field). Thus, it suffices to consider the étale case. By faithfully flat base change to a strict henselization of $R$, we are reduced to the case where our finite étale group schemes are constant. Since faithful flatness is equivalent to surjectivity (for maps between étale schemes over a base), the lemma is now physically clear.

Now we turn to the first of the two main results we want to prove. Let $\pi: J \rightarrow A$ be an optimal quotient of abelian varieties over $K$ (i.e., we assume that $\operatorname{ker} \pi$ is an abelian variety over $K$ ), and assume that $J$ has semistable reduction over $R$ (so $A$ does too). We do not yet make any hypotheses of purely toric reduction. The dual abelian varieties $A^{\vee}$ and $J^{\vee}$ again have semistable reduction, as they are isogenous to $A$ and $J$ respectively.

Theorem 8.2. With notation as above, the map $X_{J^{\vee}} \rightarrow X_{A^{\vee}}$ induced by $\pi$ is surjective.

Proof. The underlying idea comes down to two facts: Lemma 8.1 and the fact that we can lift tori on the level of $\ell$-divisible groups for any prime $\ell$. More precisely, we argue as follows. By Proposition 3.3, the map $\pi^{\vee}: A^{\vee} \rightarrow J^{\vee}$ is a closed immersion of abelian varieties. We will use this to prove that the induced map $\pi_{t}^{\vee}$ on closed fiber tori of Néron models is a closed immersion. Since the "character group" functor sets up an anti-equivalence of categories between tori over a field $F$ and finite free $\mathbf{Z}$-modules with continuous action of $\operatorname{Gal}\left(F_{s} / F\right)$, identifying closed immersions of tori with surjections of character groups and surjections of tori with "saturated injections" of character groups (i.e., injections with torsion-free cokernel), the closed immersion property for $\pi_{t}^{\vee}$ on the closed fiber tori will yield the desired surjection of character groups.

In general the "Néron model" functor doesn't behave well for closed immersions. That is, just because $\pi^{\vee}$ is a closed immersion, it does not follow purely formally that $\pi^{\vee}$ induces a closed immersion on Néron models. Nevertheless, we claim quite generally that if $B \rightarrow B^{\prime}$ is a closed immersion of abelian varieties 
over $K$ with semistable Néron models, then the induced map $T \rightarrow T^{\prime}$ on closed fiber tori is a closed immersion. For this it is sufficient to prove that the induced map on $\ell$-divisible groups $T\left[\ell^{\infty}\right] \rightarrow T^{\prime}\left[\ell^{\infty}\right]$ is a closed immersion for all primes $\ell$ (i.e., all maps $T\left[\ell^{n}\right] \rightarrow T^{\prime}\left[\ell^{n}\right]$ are closed immersions). Indeed, suppose we verify this closed immersion property on torsion, and let $H$ be the kernel of $T \rightarrow T^{\prime}$, so $H\left[\ell^{n}\right]=0$ for all primes $\ell$ and positive integers $n$. The torus $\left(H_{/ \bar{k}}^{0}\right)_{\text {red }}$ must vanish (as it has no non-trivial torsion) and hence $H$ is finite. If $N$ is the order of $H$, then $H=H[N]=0$. The map $T \rightarrow T^{\prime}$ is then a monomorphism between algebraic groups over a field and hence is a closed immersion, as desired.

In order to verify that the $\ell$-divisible group maps $T\left[\ell^{\infty}\right] \rightarrow T^{\prime}\left[\ell^{\infty}\right]$ are closed immersions for all $\ell$, we can make the faithfully flat base change to the henselization of $R$ (which commutes with formation of Néron models) to reduce to the case where $R$ is henselian. Now we recall the following basic result of Grothendieck:

Lemma 8.3. Let $R$ be a henselian local ring, $G$ a quasi-finite separated $R$ scheme of finite presentation. There is a unique decomposition

$$
G=G_{f} \coprod G^{\prime}
$$

into disjoint clopen pieces with $G_{f}$ finite over $R$ (called the "finite part" of $G$ ) and $G^{\prime}$ having empty closed fiber. The formation of $G_{f}$ is functorial in $G$ and is compatible with henselian local base change and formation of fiber products over $R$.

If moreover $G$ is a group scheme over $R$, then $G_{f}$ is a clopen subgroup scheme and there exists a unique multiplicative closed $R$-subgroup scheme $G_{\mu}$ inside of $G$ whose closed fiber is the multiplicative part of the closed fiber of $G\left(G_{\mu}\right.$ is called the "multiplicative part" of $G$ ). The formation of $G_{\mu}$ is functorial in $G$.

Proof. For the first part, see $\left[8, \mathrm{IV}_{4}, 18.5 .11(c)\right]$ (aside from the functorial properties, which are obvious). The second part, concerning group schemes, is a mechanical consequence of the first part (including the functoriality of the finite part). For example, the existence of $G_{\mu}$ follows from considering the connectedétale sequence of the Cartier dual of $G_{f}$ over the henselian local base $R$, and the uniqueness and functoriality follows from the functoriality of $G \rightsquigarrow G_{f}$ and the functoriality of the connected-étale sequence.

Remark 8.4. Assuming $R$ in Lemma 8.3 is a discrete valuation ring (with fraction field $K$ and residue field $k$ ), let us make some observations concerning the behavior of Lemma 8.3 with respect to primary components, as this will be useful later. Let's suppose that $N$ and $M$ are relatively prime integers with $N M$ divisible by the order of $G_{K}$, and hence killing $G$. Thus, by functoriality we have $G=G[N] \times{ }_{R} G[M]$ where $G[N]$ and $G[M]$ are quasi-finite separated $R$ group schemes. We claim that $G[N]$ and $G[M]$ are also flat over $R$, whence it follows that the formation of $G_{f}$ and $G_{\mu}$ is compatible with passage to "primary components". 
In other words, if $\ell$ is a prime and $\ell^{n}$ is divisible by the $\ell$-part of the order of $G_{K}$, then we claim that $G\left[\ell^{n}\right]$ is $R$-flat. From the clopen decomposition $G=G_{f} \coprod G^{\prime}$, it is easy to see that $G\left[\ell^{n}\right]=G_{f}\left[\ell^{n}\right] \coprod X_{n}$ for some finite $K$ scheme $X_{n}$, so for the issue of $R$-flatness we can replace $G$ with $G_{f}$. We are thereby reduced to the finite flat case, so we can use the proof of $[10,1.7 .2]$.

The significance of Lemma 8.3 for our purposes is the following standard consequence.

Corollary 8.5. Let $A$ be an abelian variety over the fraction field $K$ of a henselian discrete valuation ring $R$ with residue field $k$. Let $\mathcal{A}$ be the Néron model of $A$, and assume that $\mathcal{A}$ has semistable reduction. For every prime $\ell$, there exists a unique multiplicative $\ell$-divisible group $\Gamma_{\ell}$ inside of $\mathcal{A}$ whose closed fiber is the $\ell$-divisible group of the torus $T$ of $\mathcal{A}_{k}^{0}$. The formation of $\Gamma_{\ell}$ is functorial in $A$.

Proof. Fix $\ell$. By the semistability hypothesis, the multiplication maps $\ell^{n}: \mathcal{A} \rightarrow$ $\mathcal{A}$ are quasi-finite flat, so $\mathcal{A}\left[\ell^{n}\right]$ is a quasi-finite flat separated $R$-group scheme. Let $\mathcal{A}\left[\ell^{n}\right]_{\mu}$ denote its multiplicative part (as in Lemma 8.3), so the multiplicative $T\left[\ell^{n}\right] \hookrightarrow \mathcal{A}\left[\ell^{n}\right]_{k}$ lies inside of $\left(\mathcal{A}\left[\ell^{n}\right]_{\mu}\right)_{k}$. The "closed fiber" functor is an equivalence of categories between finite flat multiplicative group schemes over $R$ and $k$ (since Cartier duality reduces this to the étale case, and the "closed fiber" functor is an equivalence of categories between finite étale $R$-schemes and finite étale $k$-schemes $\left.\left[8, \mathrm{IV}_{4}, 18.5 .12\right]\right)$. Thus, there exists a unique multiplicative closed $R$-subgroup scheme $\Gamma^{(n)} \hookrightarrow \mathcal{A}\left[\ell^{n}\right]_{\mu}$ whose closed fiber is $T\left[\ell^{n}\right]$.

Moreover, using the equivalence of categories just mentioned, $\mathcal{A}\left[\ell^{n}\right]_{\mu}$ lies inside of $\mathcal{A}\left[\ell^{n+1}\right]_{\mu}$ and $\Gamma^{(n)}$ lies inside of $\Gamma^{(n+1)}$. The resulting system $\Gamma_{\ell}=\left\{\Gamma^{(n)}\right\}$ over $R$ forms an $\ell$-divisible group on the closed fiber and hence is an $\ell$-divisible group over $R$. This settles the desired existence, as well as the desired uniqueness. The functoriality of $\Gamma_{\ell}$ in $A$ follows from the functoriality of toric parts on the closed fiber of Néron models.

Returning to the proof of Theorem 8.2, recall that we were studying the map of toric parts $j_{t}: T \rightarrow T^{\prime}$ induced by a closed immersion $j: B \hookrightarrow B^{\prime}$ of semistable abelian varieties over $K$, with $R$ henselian. We wanted the map

$$
j_{t}\left[\ell^{\infty}\right]: T\left[\ell^{\infty}\right] \rightarrow T^{\prime}\left[\ell^{\infty}\right]
$$

to be a closed immersion for all primes $\ell$ (as we have seen that this forces $T \rightarrow T^{\prime}$ to be a closed immersion, which is what we really want to show). Fix $\ell$. By Corollary 8.5 there exist unique multiplicative $\ell$-divisible groups $\Gamma$ and $\Gamma^{\prime}$ over $R$ in the respective Néron models $\mathcal{B}$ and $\mathcal{B}^{\prime}$ such that $\Gamma$ and $\Gamma^{\prime}$ respectively lift the $\ell$-divisible groups of the tori of the closed fibers. Hence, it suffices to show that the $R$-map $\gamma: \Gamma \rightarrow \Gamma^{\prime}$ induced by the Néron functoriality map $\mathrm{N}(j)$ is a closed immersion. The generic fiber map $\gamma_{K}$ is a closed immersion since it "sits inside" the generic fiber $\ell$-divisible groups of $B$ and $B^{\prime}$, the map between which is a closed immersion since $j: B \rightarrow B^{\prime}$ is a closed immersion. Now we 
use Lemma 8.1 (applied at all finite torsion levels) to conclude that $\gamma$ is a closed immersion. This completes the proof of Theorem 8.2.

We now turn to a result which requires a stronger hypothesis on the closed fiber. Note that we retain the hypothesis that $R$ is henselian (this hypothesis arose in the proof of Theorem 8.2, even though it wasn't needed for the statement). Let $A$ and $B$ be abelian varieties over $K$ with purely toric reduction (i.e., their Néron models have closed fiber connected components which are tori). Let $\varphi: A \rightarrow B$ be an isogeny, and let $\varphi_{t}: T_{A} \rightarrow T_{B}$ be the induced map on the closed fiber toric parts (i.e., connected components) of the Néron models. We denote by $\varphi_{t}^{\vee}: T_{B^{\vee}} \rightarrow T_{A^{\vee}}$ the analogous map induced by the dual isogeny $\varphi^{\vee}$. Since the map $\varphi_{t}$ is an isogeny (by functoriality), the $\operatorname{kernel} \operatorname{ker}\left(\varphi_{t}\right)$ is a finite multiplicative $k$-group scheme.

For any finite multiplicative $k$-group scheme $G$, we let $\widetilde{G}$ denote the (unique) multiplicative finite flat $R$-group scheme with closed fiber $G$. For example, $\widetilde{\operatorname{ker}\left(\varphi_{t}\right)}$ is a multiplicative $R$-group scheme which lies inside of

$$
\operatorname{ker}(\mathrm{N}(\varphi))_{\mu}
$$

(where $\mathrm{N}(\varphi)$ is the map induced by Néron functoriality). Thus, we have a natural closed immersion

$$
\left.\widetilde{\left(\operatorname{ker} \varphi_{t}\right.}\right)_{K} \hookrightarrow \operatorname{ker} \varphi
$$

and likewise we have a natural quotient map

$$
\operatorname{ker}\left(\varphi^{\vee}\right)^{\vee} \rightarrow \widetilde{\operatorname{ker}\left(\varphi_{t}^{\vee}\right)_{K}^{\vee}}
$$

dual to the natural closed immersion using the isogeny $\varphi^{\vee}$.

By the duality theory for abelian varieties (particularly the adjointness of $\varphi$ and $\varphi^{\vee}$ with respect to the scheme-theoretic Weil pairing over $\left.K\right)$, there is a canonical perfect duality $K$-group scheme duality between $\operatorname{ker}(\varphi)$ and $\operatorname{ker}\left(\varphi^{\vee}\right)$ over $K$, whence there is a natural quotient map of $K$-group schemes

$$
\operatorname{ker}(\varphi) \simeq \operatorname{ker}\left(\varphi^{\vee}\right)^{\vee} \rightarrow \widetilde{\operatorname{ker}\left(\varphi_{t}^{\vee}\right)_{K}^{\vee}}
$$

Theorem 8.6. The diagram of K-group schemes

$$
0 \rightarrow \widetilde{\operatorname{ker}\left(\varphi_{t}\right)_{K}} \rightarrow \operatorname{ker}(\varphi) \rightarrow \widetilde{\operatorname{ker}\left(\varphi_{t}^{\vee}\right)_{K}^{\vee} \rightarrow 0}
$$

is exact.

The content of the proof is the Grothendieck Orthogonality Theorem. Moreover, Theorem 8.6 is implicit in Grothendieck's construction of the monodromy pairing for semiabelian varieties.

Proof. The exact sequence of the theorem says that the finite flat $K$-group schemes

$$
\left.\operatorname{ker}(\varphi) / \widetilde{\operatorname{ker}\left(\varphi_{t}\right)_{K}} \quad \text { and } \quad \widetilde{\operatorname{ker}\left(\varphi_{t}^{\vee}\right.}\right)_{K}
$$


are canonically Cartier dual to each other compatibly with the perfect duality between $\operatorname{ker}(\varphi)$ and $\operatorname{ker}\left(\varphi^{\vee}\right)$. More precisely, let $\mathcal{A}$ and $\mathcal{B}$ denote the Néron models of $A$ and $B$, respectively, let

$$
G=\operatorname{ker}(\mathcal{A} \rightarrow \mathcal{B}), \quad G^{\vee}=\operatorname{ker}\left(\mathcal{B}^{\vee} \rightarrow \mathcal{A}^{\vee}\right),
$$

so $G$ and $G^{\vee}$ are both quasi-finite flat separated $R$-group schemes whose generic fibers are the $\operatorname{ker}(\varphi)$ and $\operatorname{ker}\left(\varphi^{\vee}\right)$ in the theorem (the $R$-flatness of $G$ and $G^{\vee}$ arises from the semiabelian condition, since any quasi-finite morphism between semi-abelian schemes is necessarily flat, as can be checked on geometric fibers). Being quasi-finite flat and separated, the $R$-group schemes $G$ and $G^{\vee}$ have canonical respective "finite parts" $G_{f}$ and $G_{f}^{\vee}$ and "multiplicative parts" $G_{\mu}$ and $G_{\mu}^{\vee}$ (as in Lemma 8.3). Beware that we do not claim $G_{f}^{\vee}\left(\right.$ resp. $G_{\mu}^{\vee}$ ) is the Cartier dual to $G_{f}$ (resp. $G_{\mu}$ ); usually such duality does not hold.

Since $G_{\mu}$ and $G_{\mu}^{\vee}$ are finite flat $R$-group schemes, the quotients $G / G_{\mu}$ and $G^{\vee} / G_{\mu}^{\vee}$ make sense as quasi-finite flat separated $R$-group schemes. The theorem almost says that there is a canonical duality between $\left(G / G_{\mu}\right)_{K}=G_{K} /\left(G_{\mu}\right)_{K}$ and $\left(G_{\mu}^{\vee}\right)_{K}$, induced by the duality between $G_{K}=\operatorname{ker}(\varphi)$ and $G_{K}^{\vee}=\operatorname{ker}\left(\varphi^{\vee}\right)$, except for the mild problem that $G_{\mu}$ might be larger than $\widetilde{\operatorname{ker}\left(\varphi_{t}\right)}$ (i.e., possibly $\left(G_{\mu}\right)_{k}$ is not entirely inside of $\left.\mathcal{A}_{k}^{0}\right)$ and likewise $G_{\mu}^{\vee}$ might be larger than $\widetilde{\operatorname{ker}\left(\varphi_{t}^{\vee}\right)}$.

We will work on $\ell$-primary components for each prime $\ell$ individually. In order to permit this, we use Remark 8.4. We will first treat the more subtle case when $\ell$ is the residue characteristic, and then we'll handle the case when it isn't. The advantage of working with the case in which $\ell$ is the residue characteristic is that multiplicative finite $k$-group schemes are automatically connected. Thus, in this case $\left(G_{\mu}\right)_{\ell}=\widetilde{\operatorname{ker}\left(\varphi_{t}\right)_{\ell}}$ and $\left.\left(G_{\mu}^{\vee}\right)_{\ell}=\widetilde{\operatorname{ker}\left(\varphi_{t}^{\vee}\right.}\right)_{\ell}$.

Since

$$
0 \rightarrow G_{K} \rightarrow A \rightarrow B \rightarrow 0
$$

is an exact sequence of abelian sheaves on the fppf site over $\operatorname{Spec}(K)$, by the usual snake lemma argument (and the fact that the $\ell$-part $\left(G_{K}\right)_{\ell}$ of $G_{K}$ is killed by a big power of $\ell$ ) we obtain an exact sequence

$$
0 \rightarrow\left(G_{K}\right)_{\ell} \rightarrow A\left[\ell^{\infty}\right] \rightarrow B\left[\ell^{\infty}\right] \rightarrow 0 .
$$

Arguing as in Corollary 8.5, there is an exact sequence over $\operatorname{Spec}(R)$

$$
0 \rightarrow\left(G_{\mu}\right)_{\ell} \rightarrow \mathcal{A}\left[\ell^{\infty}\right]_{t} \rightarrow \mathcal{B}\left[\ell^{\infty}\right]_{t} \rightarrow 0
$$

which lifts the exact sequence involving $\ell$-divisible groups of tori on the closed fiber (as $\left(G_{\mu}\right)_{\ell}$ must be in the relative connected component of $\mathcal{A}$ ). Passing to the generic fiber over $K$ gives us a commutative diagram with exact rows and closed immersions along columns

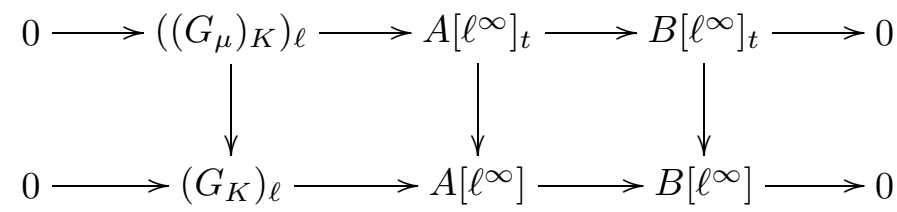


where $A\left[\ell^{\infty}\right]_{t}$ denotes the $K$-fiber of the $\ell$-divisible group $\left\{\mathcal{A}\left[\ell^{n}\right]_{t}\right\}$, and likewise for $B\left[\ell^{\infty}\right]_{t}$.

Using the snake lemma in the category of fppf abelian sheaves over $\operatorname{Spec}(K)$, we get a short exact sequence of cokernels

$$
0 \rightarrow\left(\left(G / G_{\mu}\right)_{K}\right)_{\ell} \rightarrow A\left[\ell^{\infty}\right] / \text { (toric) } \rightarrow B\left[\ell^{\infty}\right] /(\text { toric }) \rightarrow 0
$$

where all maps are the natural ones, and right two terms are $\ell$-divisible groups over $K$.

The Grothendieck Orthogonality Theorem (see [9, Exp. IX, Prop 5.6]) asserts that the perfect scheme-theoretic Weil pairing between $A\left[\ell^{n}\right]$ and $A^{\vee}\left[\ell^{n}\right]$ makes $A\left[\ell^{n}\right]_{t}$ and $A^{\vee}\left[\ell^{n}\right]_{f}$ exact annhilators, where $A\left[\ell^{n}\right]_{f}$ denotes the $K$-fiber of the finite part of the $\mathcal{A}^{0}\left[\ell^{n}\right]$ and $A\left[\ell^{n}\right]_{t}$ denotes the $K$-fiber of the unique $R$-subgroup scheme in $\mathcal{A}^{0}\left[\ell^{n}\right]$ lifting the $\ell^{n}$-torsion on the closed fiber torus. By the purely toric condition applied to $A^{\vee}$, we see $A^{\vee}\left[\ell^{n}\right]_{f}=A^{\vee}\left[\ell^{n}\right]_{t}$. Thus, the orthogonality theorem says that $A\left[\ell^{n}\right] / A\left[\ell^{n}\right]_{t}$ and $A^{\vee}\left[\ell^{n}\right]_{t}$ are in perfect duality via the schemetheoretic Weil pairing over $K$.

Passing to the limit, we get a canonical isomorphism of $\ell$-divisible groups

$$
A\left[\ell^{\infty}\right] /(\text { toric })=\left(A^{\vee}\left[\ell^{\infty}\right]_{t}\right)^{\vee} .
$$

But $\varphi$ and $\varphi^{\vee}$ are adjoint with respect to Weil pairing, so we conclude that the diagram

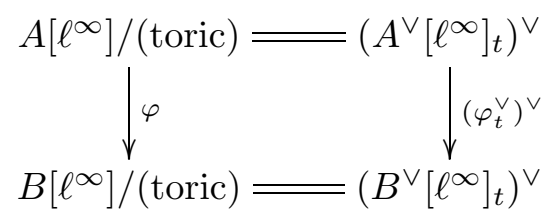

commutes. Thus, we get an isomorphism between the kernels of these vertical isogenies. The kernel of the left column is $\left(\left(G / G_{\mu}\right)_{K}\right)_{\ell}$, as we saw above. Meanwhile, the kernel of the right is (by duality theory of $\ell$-divisible groups) exactly the dual of $\operatorname{ker}\left(\varphi_{t}^{\vee}\right)=\left(G_{\mu}^{\vee}\right)_{K}$. This gives the desired perfect duality between $\left(G / G_{\mu}\right)_{K}$ and $\left(G_{\mu}^{\vee}\right)_{K}$ on $\ell$-primary parts for $\ell$ equal to the residue characteristic.

Now we consider the case when $\ell$ is not equal to the residue characteristic. There is no loss of generality in passing to the case of a strictly henselian base $R$. Thus, the closed fiber tori have constant $\ell$-divisible groups. Also, we can

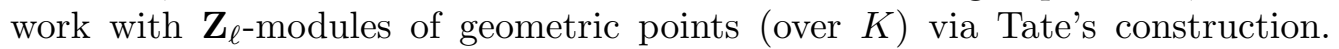
The "toric" part of the $\ell$-adic Tate module $T_{\ell}(A)$ is exactly the (saturated) maximal submodule with trivial Galois action, since a compatible system of $\ell$ power torsion points in $A(K)=\mathcal{A}(R)$ must lie entirely inside of $\mathcal{A}^{0}(R)$ (thanks to the finiteness of the component group) and we can identify $\mathcal{A}^{0}(R)\left[\ell^{n}\right]$ with the (constant) $\ell^{n}$-torsion on the split torus $\mathcal{A}_{k}^{0}$ over the separably closed $k$.

Using inverse limits, we see that $T_{\ell}(A) \rightarrow T_{\ell}(B)$ is injective with cokernel $\left(G_{K}\right)_{\ell}$ (= geometric points of $\ell$-part), and this cokernel is exactly $\operatorname{ker}(\varphi)_{\ell}$. Likewise, the cokernel of the map

$$
T_{\ell}(A)_{t} \rightarrow T_{\ell}(B)_{t}
$$


on "toric" parts (i.e., $\ell$-adic Tate module generic fibers of the lifts of the $\ell$ divisible groups of closed fiber tori) is $\left(\widetilde{\operatorname{ker}\left(\varphi_{t}\right)_{K}}\right)_{\ell}$.

Thus, we get a commutative diagram with horizontal exact sequences

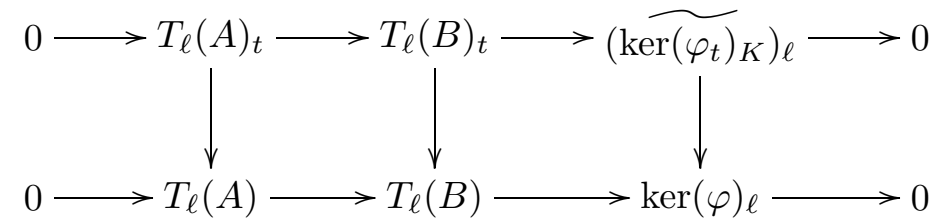

with columns given by the natural maps. These vertical maps are all injective, so by the snake lemma we get a short exact sequence of cokernels. We can now use the exact same Weil pairing arguments with the Grothendieck orthogonality theorem (now in the easier " $\ell \neq p$ " form of the orthogonality theorem $[9$, Exp IX, 2.4]), essentially just as we argued in the previous case. One distinction is that the use of inverse limit Tate modules rather than direct limits causes some maps to switch direction.

More specifically we have a commutative square with horizontal isomporphisms (thanks to the orthogonality theorem)

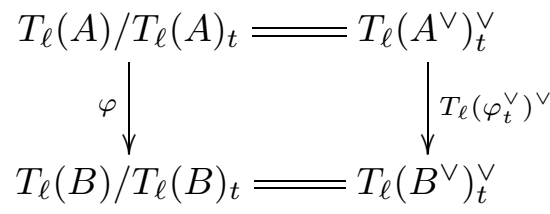

This induces an isomorphism

$$
\left.\left.\left((\operatorname{ker} \varphi) / \widetilde{\operatorname{ker}\left(\varphi_{t}\right.}\right)_{K}\right)_{\ell} \simeq\left(\widetilde{\left(\operatorname{ker} \varphi_{t}^{\vee}\right.}\right)_{K}\right)_{\ell}^{\vee}
$$

between the vertical cokernels, and by construction this isomorphism is compatible with Weil pairings, whence the desired perfect pairing has been shown.

Let $R$ be an arbitrary dvr (not necessarily henselian), let $\varphi: A \rightarrow B$ be an isogeny, and let $\varphi_{t}: T_{A} \rightarrow T_{B}$ be the induced map on the closed fiber toric parts, as above.

Corollary 8.7. The order of $\operatorname{ker}(\varphi)$ is the product of the orders of $\operatorname{ker} \varphi_{t}$ and $\operatorname{ker} \varphi_{t}^{\vee}$.

Proof. Pass to the henselization of $R$ and use Theorem 8.6.

\section{Acknowledgements}

This paper was inspired by lectures of R. Coleman and K. Ribet, and a letter from Ribet to Mestre (see [17]), which contains some of the results of the present paper in the case when $A$ has dimension 1. The second author would like to thank A. Abbes, A. Agashe, D. Kohel, and D. Lorenzini, for helpful conversations. 


\section{References}

[1] A. Agashe and W. A. Stein, Visibility of Shafarevich-Tate groups of abelian varieties: Evidence for the Birch and Swinnerton-Dyer conjecture, (2001).

[2] S. Bosch, W. Lütkebohmert, and M. Raynaud, Néron models. Ergebnisse der Mathematik und ihrer Grenzgebiete (3), 21. Springer-Verlag, Berlin, 1990.

[3] C. Chevalley, Une démonstration d'un théorème sur les groupes algébriques, J. Math. Pures Appl. (9) 39 1960, 307-317.

[4] B. Conrad, A modern proof of Chevalley's theorem on algebraic groups, http://www-math.mit.edu/ dejong/papers/chev.dvi

[5] B. Edixhoven, L'action de l'algèbre de Hecke sur les groupes de composantes des jacobiennes des courbes modulaires est "Eisenstein". Courbes modulaires et courbes de Shimura (Orsay, 1987/1988). Astérisque No. 196-197, (1991), 7-8, 159-170 (1992).

[6] M. Emerton, Optimal quotients of modular Jacobians, (2001), preprint.

[7] E. V. Flynn, F. Leprévost, E. F. Schaefer, W. A. Stein, M. Stoll, and J. L. Wetherell, Empirical evidence for the Birch and Swinnerton-Dyer conjectures for modular Jacobians of genus 2 curves, Math. Comp. 70 (2001), no. 236, 1675-1697 (electronic).

[8] A. Grothendieck, Éléments de géométrie algébrique, Publications Mathématiques IHES, $\mathbf{4 , 8 , 1 1 , 1 7 , 2 0 , 2 4 , 2 8 , 3 2 , ~ 1 9 6 0 - 7 . ~}$

[9] A. Grothendieck, Groupes de monodromie en géométrie algébrique, Lecture Notes in Math 288, Springer-Verlag, Heidelberg (1972).

[10] N. Katz, B. Mazur, Arithmetic moduli of elliptic curves. Annals of Mathematics Studies, 108. Princeton University Press, Princeton, NJ, 1985.

[11] D. R. Kohel, Hecke module structure of quaternions. Class field theory - its centenary and prospect (Tokyo, 1998), 177-195, Adv. Stud. Pure Math., 30, Math. Soc. Japan, Tokyo, 2001.

[12] D. R. Kohel and W. A. Stein, Component Groups of Quotients of $J_{0}(N)$, Proceedings of the 4th International Symposium (ANTS-IV), Leiden, Netherlands, July 2-7, 2000 (Berlin), Springer, 2000.

[13] B. Mazur, Modular curves and the Eisenstein ideal. Inst. Hautes Études Sci. Publ. Math. No. 47 (1977), 33-186 (1978).

[14] J.-F. Mestre, La méthode des graphes. Exemples et applications. Proceedings of the international conference on class numbers and fundamental units of algebraic number fields (Katata, 1986), 217-242, Nagoya Univ., Nagoya, 1986.

[15] J.-F. Mestre and J. Oesterlé, Courbes de Weil semi-stables de discriminant une puissance $m$-ième, J. Reine Angew. Math. 400 (1989), 173-184.

[16] D. Mumford, Abelian varieties. Tata Institute of Fundamental Research Studies in Mathematics, No. 5, Published for the Tata Institute of Fundamental Research, Bombay; Oxford University Press, London 1970.

[17] K. A. Ribet, Letter about component groups of elliptic curves, arXiv:math.AG/0105124v1 (2001).

[18] J. Tate, Algorithm for determining the type of a singular fiber in an elliptic pencil. Modular functions of one variable, IV (Proc. Internat. Summer School, Univ. Antwerp, Antwerp, 1972), 33-52. Lecture Notes in Math., Vol. 476, Springer, Berlin, 1975.

Department of Mathematics, University of Michigan, 2074 East Hall, Ann Arbor, MI 48109-1109, USA.

E-mail address: bdconrad@math.1sa.umich.edu

Harvard University, Department of Mathematics, One Oxford Street, Cambridge, MA 02138, USA.

E-mail address: was@math.harvard.edu 